\title{
Solid-state titania-based gas sensor for liquefied petroleum gas detection at room temperature
}

\author{
B C YADAV*, ANURADHA YADAV, TRIPTI SHUKLA and SATYENDRA SINGH \\ Nanomaterials and Sensors Research Laboratory, Department of Physics, University of Lucknow, \\ Lucknow 226007 , India
}

MS received 27 May 2010; revised 21 July 2010

\begin{abstract}
This paper reports the liquefied petroleum gas (LPG) sensing of titanium dioxide (Qualigens, India). Scanning electron micrographs and X-ray diffraction studies of samples were done. SEM shows that the material is porous and has grapes-like morphology before exposure to the LPG. XRD patterns reveal the crystalline nature of the material. The crystallites sizes of the $\mathrm{TiO}_{2}$ were found in the range of $30-75 \mathrm{~nm}$. Variations in resistance with exposure of LPG to the sensing element were observed. The average sensitivity for different volume percentages of gas was estimated. The maximum value of average sensitivity was 1.7 for higher vol.\% of LPG. Percentage sensor response $(\% \mathrm{SR})$ as a function of time was calculated and its maximum value was $45 \%$. Response time of the sensor was $70 \mathrm{~s}$. The sensor was quite sensitive to LPG and results were found reproducible.
\end{abstract}

Keywords. LPG sensor; $\mathrm{TiO}_{2}$; $\mathrm{SEM}$; XRD; sensitivity.

\section{Introduction}

There is an increasing interest to find new materials in order to develop high-performance solid-state gas sensors. Semiconductor metal oxide sensors are alternative for inexpensive and robust detection systems. Semiconducting metal oxide material such as $\mathrm{ZnO}, \mathrm{TiO}_{2}, \mathrm{SnO}_{2}$, $\mathrm{In}_{2} \mathrm{O}_{3}$, etc., play an important role in almost all fields of electronics (Yadav et al 2008, 2009), especially their applications to gas sensors, which require controlled nanosize of the sensing materials.

$\mathrm{TiO}_{2}$ is an important oxide material for broad range of gas-sensing applications (Yadav et al 2007; More et al 2009) because of its surface chemistry, charge transport and electrical properties (Carney et al 2005; Manera et al 2007). It is a versatile material widely used in industry, research and environmental cleaning. Physical properties of $\mathrm{TiO}_{2}$ have given various fields of investigation to researchers, which make it suitable for various applications (Lee et al 2000; Devi et al 2002; Coronado et al 2003; Faramarz et al 2005; Dhawale et al 2008; Thiagarajan et al 2009). The first titania gas sensor was developed in the late 1970s and early 1980s, and primarily used to detect the stoichiometric air to fuel ratio (A/F) (Micheli 1984). It was also used to detect a wide variety of gaseous species such as $\mathrm{O}_{2}$ (Kirner et al 1990; Li and Chen 1996), $\mathrm{H}_{2}$ (Birkefeld et al 1992), $\mathrm{CO}$ (Bonini et al 2000) and $\mathrm{NO}_{x}$ (Guidi et al 1999). $\mathrm{TiO}_{2}$ powder can exist in the

*Author for correspondence (balchandra_yadav@rediffmail.com) anatase, rutile or brookite phase, with the most stable form being rutile, to which the others will convert at sufficiently high temperatures. The brookite phase has an orthorhombic crystal structure, and although similar to rutile in its mechanical properties, it is rarely used commercially. In contrast, the anatase and rutile forms are tetragonal systems that have found wide applications $(\mathrm{Wu}$ 2009). Among these, the anatase phase is known to exhibit better gas-sensing behaviour where as rutile is the most stable phase. Even though the energy band gap $(3 \cdot 239 \mathrm{eV})$ of the anatase phase is wider than that of the rutile $(3.02 \mathrm{eV})$ structure, recombination of electrons and holes occurs much faster on surface of rutile phase. Since the gas-sensing behaviour is mostly confined to the surface of the sensing material, its surface area must be increased to maximize this gas-sensing behaviour (Baruwati et al 2006; Shinde et al 2007).

\section{Experimental}

The starting material was $\mathrm{TiO}_{2}$ (Qualigens, 98\% pure) and glass powder. Glass powder was used as binder. These were made fine on grinding in mortar with pestle for 6-8 h. The pelletization of this material having dimensions $9 \mathrm{~mm}$ in diameter and $5 \mathrm{~mm}$ in thickness was done by using hydraulic press (M.B. Instruments, Delhi, India) under an uniaxial pressure of $462 \mathrm{MPa}$ at room temperature. This pellet was heat-treated in an electric furnace (Ambassador, India) at $400^{\circ} \mathrm{C}$ for $3 \mathrm{~h}$, and after annealing it was exposed to liquefied petroleum gas (LPG) in 

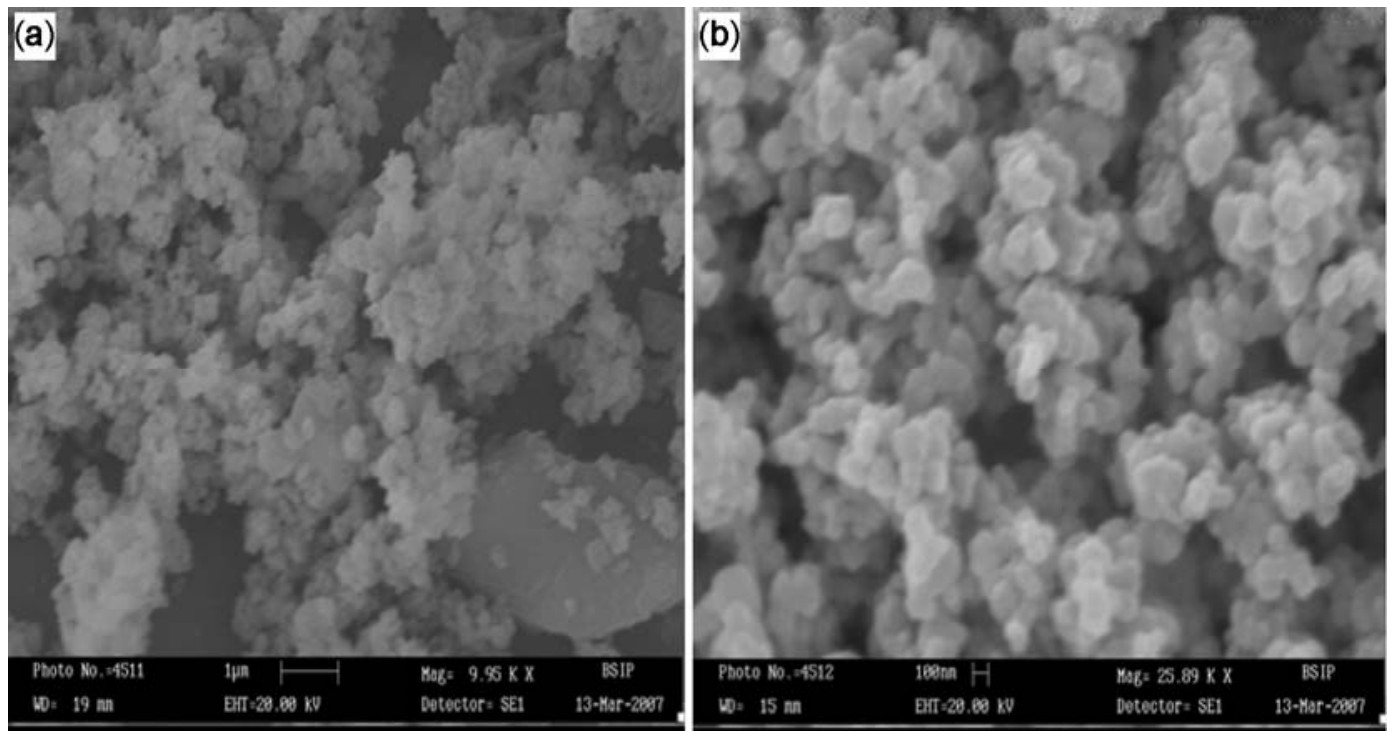

Figure 1. SEM of $\mathrm{TiO}_{2}$ in form of pellet: (a) at microscale and (b) at nanoscale.

a specially designed gas chamber in controlled conditions. The corresponding variations in resistance with the time were recorded by using a digital multimeter.

\section{Characterizations of synthesized material}

\subsection{Surface morphology}

Surface morphology of sensing element was studied using scanning electron microscope unit (SEM, LEO0430, Cambridge). Scanning electron micrographs of the sensing element annealed at $400^{\circ} \mathrm{C}$ are shown in figure 1a on microscale and at low magnification $(9.95 \mathrm{KX})$, and figure $1 \mathrm{~b}$ on nanoscale and at high magnification (25.89 KX). SEM shows that crystallites of $\mathrm{TiO}_{2}$ combining with adhesive glass particles form beautiful grapes-like clusters, leaving more spaces as pores.

Higher porosity increases the surface-to-volume ratio of the materials, which in turn increases the diffusion rate of gas and therefore helps in getting good sensitivity. SEMs show that the crystallites are agglomerated, uniform in size and equally distributed.

\section{$3.2 \quad X$-ray diffraction}

$\mathrm{X}$-Ray diffraction pattern shown in figure 2 obtained by X-Pert, PRO XRD system (Netherland) reveals crystalline nature of the sample. The average crystallite size of the sensing material was calculated by Debye-Scherer formula, which is as follows:

$$
D=\frac{K \lambda}{\beta \cos \theta}
$$

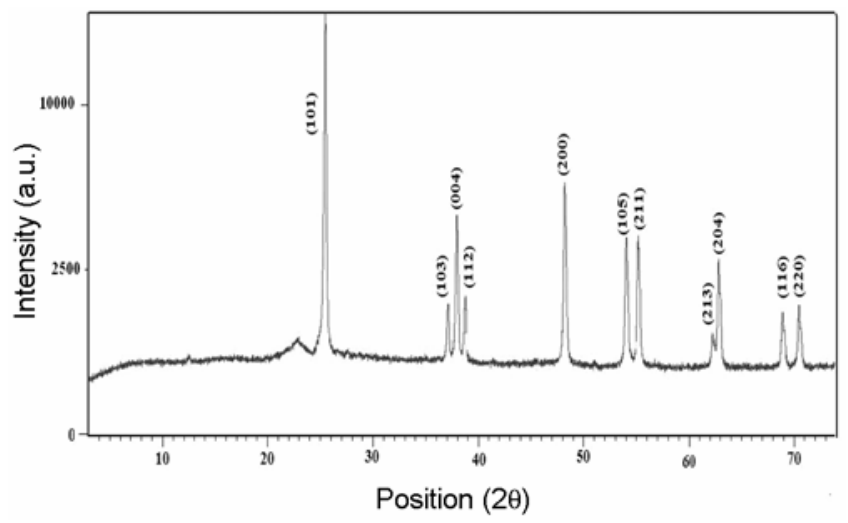

Figure 2. XRD pattern of sensing material in the form of powder.

where $D$ is the average size of crystallite assuming it to be cubic three dimensional, $K=0.94, \lambda$ the wavelength of $\mathrm{X}$-ray radiation having value $1.5418 \AA, \beta$ the full width at half maximum (FWHM) of the diffraction peak and $\theta$ the angle of diffraction.

The XRD pattern shows a high-intensity peak, centered at $2 \theta=25^{\circ}$, assigned to tetragonal $\mathrm{TiO}_{2}$ (101) reflection having $d$ spacing $3.5180 \AA$ And FWHM $0 \cdot 17^{\circ}$. Also, a peak with a low intensity at $2 \theta=70^{\circ}$ is assigned to $\mathrm{TiO}_{2}(220)$ reflection having $d$ spacing $1.33473 \AA$ and FWHM $0 \cdot 4^{\circ}$. The other intense peak is at $2 \theta=38^{\circ}$ with $d$ spacing and FWHM $2.37997 \AA$ and $0.33^{\circ}$, respectively corresponding to plane (112). Other higher angle of reflections such as (200) and (211) were indicating crystalline nature of $\mathrm{TiO}_{2}$. The XRD data thus, confirm the formation of $\mathrm{TiO}_{2}$. The minimum crystallite size of the $\mathrm{TiO}_{2}$ was $30 \mathrm{~nm}$ corresponding to reflection (105) with $d$ 
spacing and FWHM $1 \cdot 70034 \AA$ and $0 \cdot 315^{\circ}$, respectively, indicating its nanocrystalline behaviour.

\section{Gas-sensing characteristic}

The pellet of the sensing element is subjected to exposition of LPG. Variations in resistance have been taken with the variation of the time in seconds. The gas sensitivity is defined as

$$
S=\frac{R_{\mathrm{air}}}{R_{\mathrm{gas}}},
$$

where $R_{\text {air }}$ and $R_{\text {gas }}$ stand for the resistance of the sensor in air and in the sample gas, respectively (Srivastava et al 2006).

Sensor response of a sensing material (Yadav et al $2008,2009)$ is defined as

$$
\% \mathrm{SR}=\frac{\left|R_{\mathrm{a}}-R_{\mathrm{g}}\right|}{R_{\mathrm{a}}} \times 100
$$

\section{Results and discussion}

During experiment, every time, prior to exposing the LPG to $\mathrm{TiO}_{2}$ pellet, it was allowed to equilibrate inside the gas chamber at room temperature for $20-25 \mathrm{~min}$ and the stabilized resistance was taken as $R_{\mathrm{a}}$. The stabilization of the sensing element in ambient air is important because it ensures the stable zero level for gas-sensing applications. The variations in resistance with the exposure time for different vol.\% of LPG were observed and are shown in

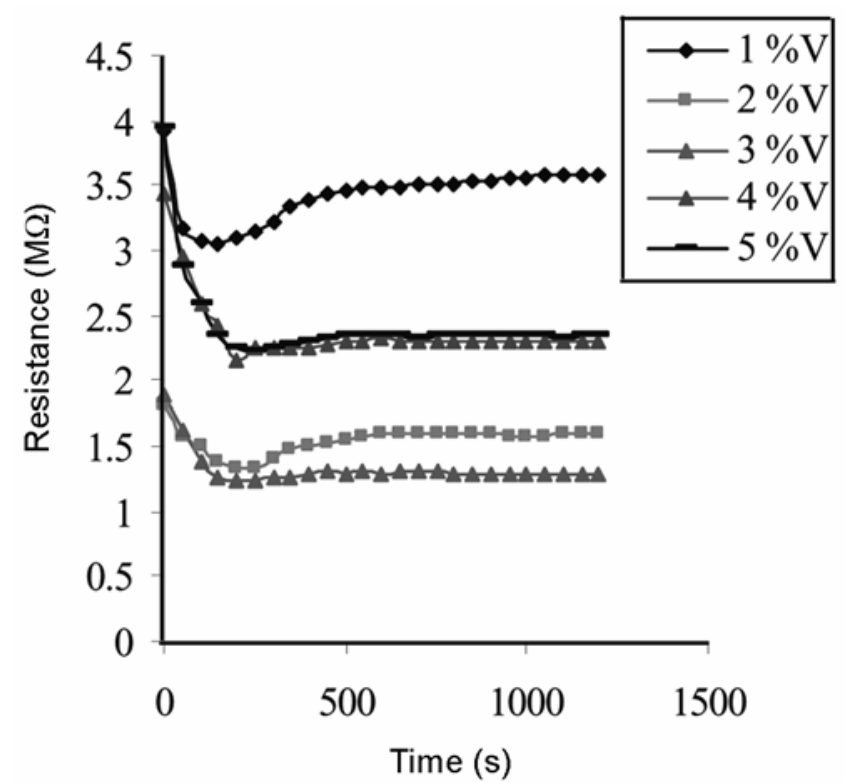

Figure 3. Resistance of sensing material vs time after exposure. figure 3. Due to $n$-type behaviour of $\mathrm{TiO}_{2}$, the electrical conductivity increases with reducing gas like LPG, and hence, the resistance of the sensing material decreases with time of exposure to the gas. It is evinced from figure 3 that the sensor resistance decreased rapidly within a few seconds and then increased and further exhibited a stable value. More et al 2008 observed similar behaviour for $\mathrm{TiO}_{2}$ films after annealing at $698 \mathrm{~K}$. The variation of gas sensitivity with time is shown in figure 4 . The maximum sensitivity was 1.8 for 5 vol.\% of LPG. It has an average gas sensitivity of 1.7 for $5 \mathrm{vol} . \%$ of LPG, as shown in

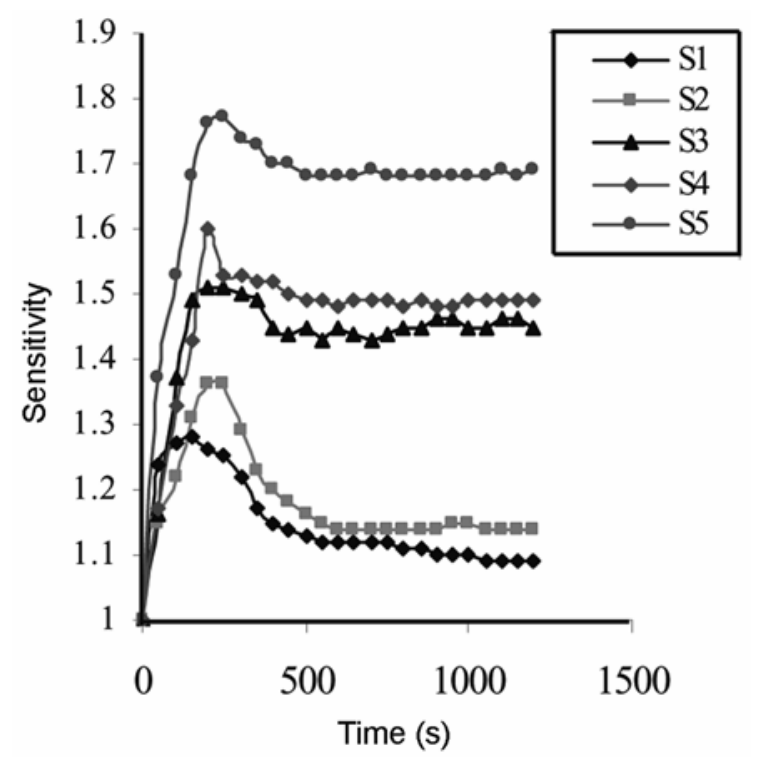

Figure 4. Sensitivity of the sensing material vs time after exposure.

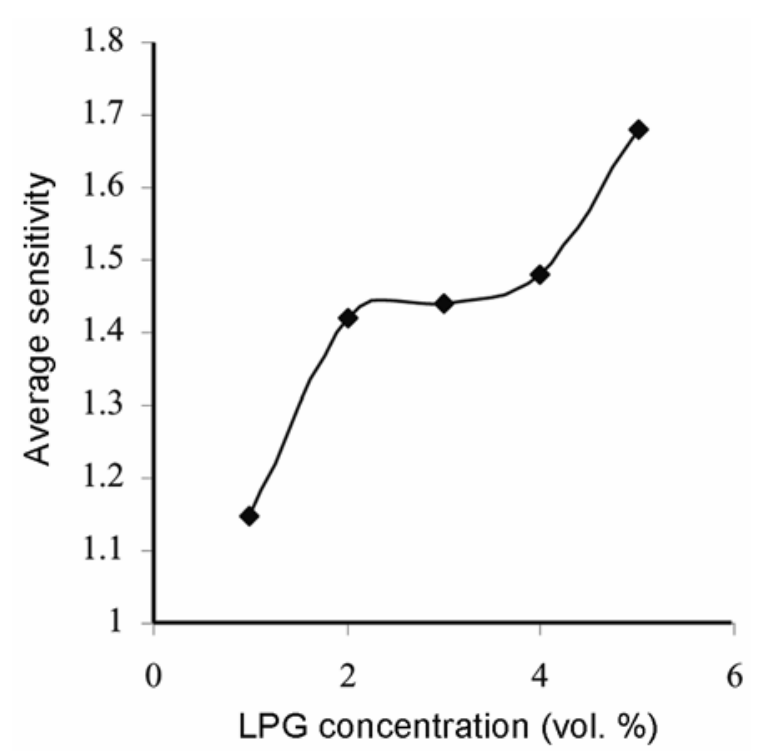

Figure 5. Average sensitivity of sensing material vs concentration of LPG. 
figure 5. Gas response curves drawn in figure 6 show that when the LPG was introduced in the gas chamber, the response increased with time during which the gas was exposed. Response time is the time during which the sensor attains $90 \%$ of its maximum response. Its value for the sensing element was $70 \mathrm{~s}$. Figure 7 shows the variation of resistance with temperature. At a higher temperature range, as the temperature increases, the resistance of the sensing material decreases. This shows the $n$-type behaviour of the sensing material (Chen et al 2008). The Arrhenius plot shown in figure 8 presents the variations of logarithmic resistance as a function of inverse temperature. This establishes the semiconducting nature

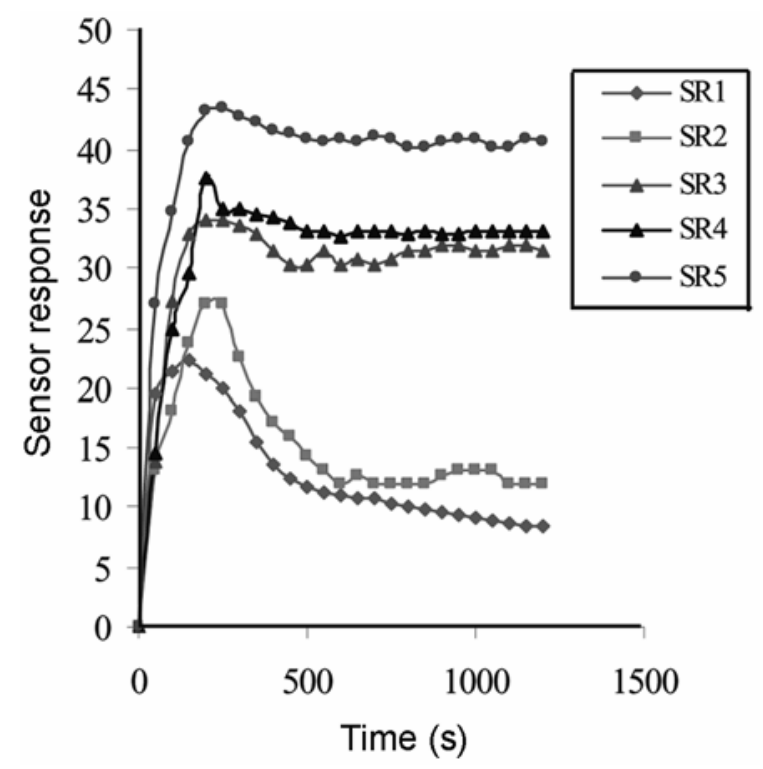

Figure 6. Sensor response vs time after exposure for sensing material.

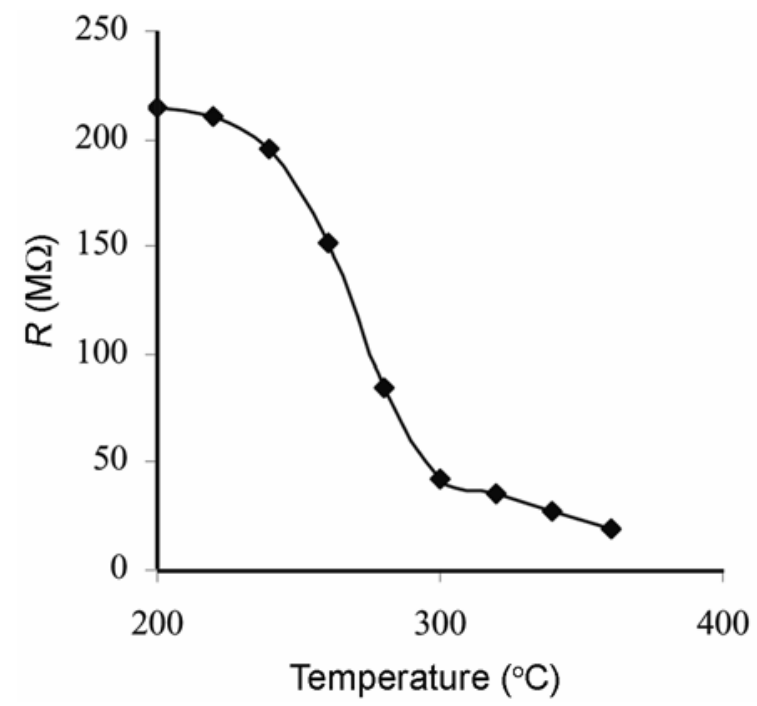

Figure 7. Resistance vs temperature for the sensing element. of the material which is due to the thermally activated mobility of carriers rather than to a thermally activated generation of these. The activation energy determined from the Arrhenius plot was $0.93 \mathrm{eV}$. Figure 9 shows the reproducibility curve for higher volume, i.e. 5 vol. $\%$ of LPG. The sensor is reproducible within $\pm 95 \%$ accuracy. The ageing effect on the sensing material was investigated and is plotted in figure 10. It was observed that the performance of device was affected by $\sim 10 \%$ after 3 months.

The principle of operation of the semiconductor gas sensor is based on the interaction of gas molecules with the surface, which produces an interchange or trapping of free charge carriers (Liu et al 2008). This sensing

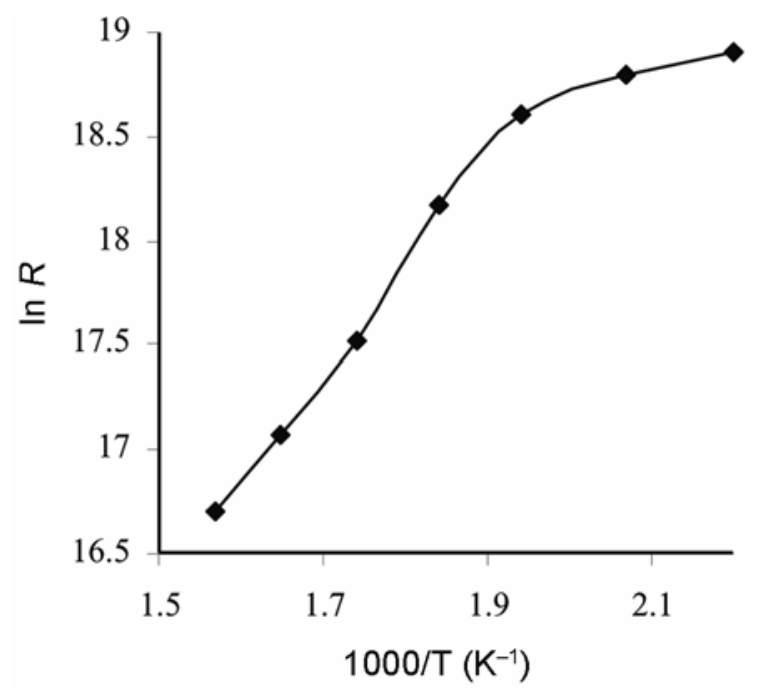

Figure 8. Arrhenius plot for sensing element.

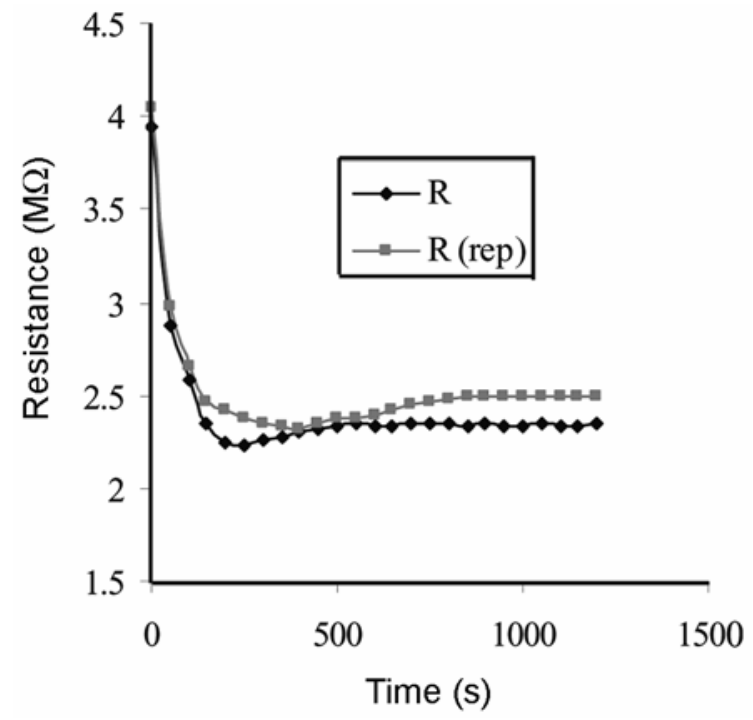

Figure 9. Reproducibility curve for sensing element exposed with 5 vol.\% LPG. 
mechanism implies that the surface of the material is extremely important from the basic point of view. Three key factors have been recognized to control the sensor response, i.e. the receptor function, the transducer function and the utility factor. The receptor function is supplied either by the surface of the grain or by a foreign material dispersed on them (Cabot et al 2000; Ruiz et al 2005). The transducer function is related to the grain boundaries and intensified extraordinarily when the grain size becomes smaller than twice the thickness of the space charge layer (Kitamura and Yokoyama 1991; Barsan et al 2007). The utility factor is the ratio of the grains accessible for the target gas. Thus, obtaining small particles would improve the sensing efficiency. In the case of $\mathrm{TiO}_{2}$, these parameters can be effectively controlled by hydrothermal treatment.

The electrical characteristics of $\mathrm{TiO}_{2}$ critically depend on the chemical composition of the surface and work function. In our experiment, surface sites of the $\mathrm{TiO}_{2}$ and the electron acceptor properties, the adsorption, the surface reaction and subsequent desorption of the LPG are key factors to determine its performance.

Metal oxide semiconductors are mainly used to detect small concentrations of reducing and combustible gases in air. In the absence of LPG, $\mathrm{O}_{2}^{-}$gets adsorbed on the surface of $\mathrm{TiO}_{2}$ pellet and it extracts electrons from the conduction band creating a depletion layer at the surface of the individual grains and intragranular region. Thus, equilibrium of the chemisorption process results in the stabilization of the surface resistance. When we expose the LPG to the surface area of semiconducting oxide, reaction takes place between surface molecules and LPG. The interaction mechanism between the gas phase and the sensing material involves mainly physiosorption, chemisorption, surface defects and bulk defects.

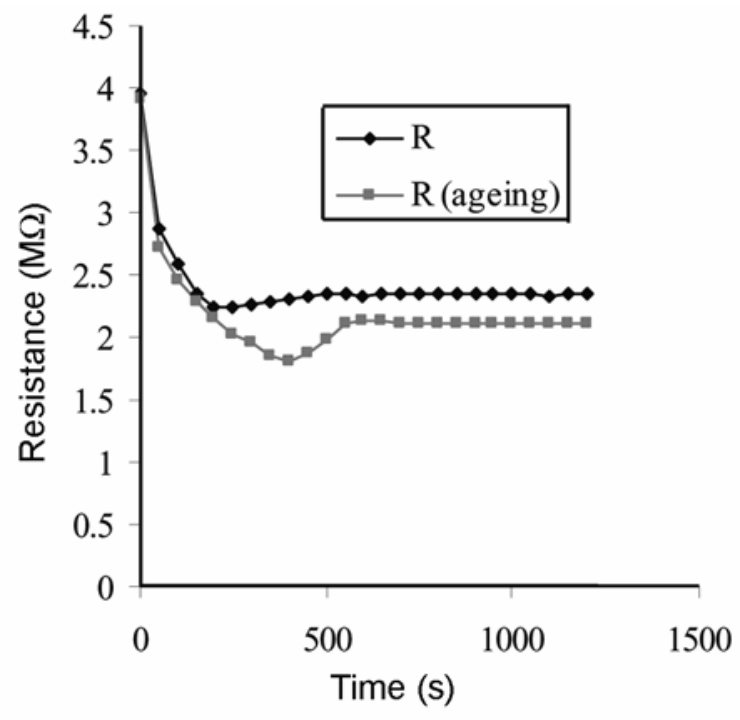

Figure 10. Ageing effect after 3 months for sensing element exposed with 5 vol. $\%$ LPG.
Physisorption is the weakest form of adsorption to solid surface and is essentially maintained by the van der Waal interaction. On the other hand, chemisorption is a stronger interaction that shows a better sensitivity and selectivity. In this kind of interactions adsorbate forms chemical bonds with the surface atoms and thus the electronic structure of both the adsorbate and the surface are modified. Chemisorption is usually promoted by surface defects. For these sensors, the defect chemistry of the oxide is very important because the bulk of the material must equilibrate with the oxygen in the system. The change in electrical conductivity of the material has been represented by the following equation

$$
\sigma \sim \sigma_{0} \exp \left(E_{\mathrm{a}} / k T\right) p\left(\mathrm{O}_{2}\right)^{1 / n},
$$

where $\sigma$ is the electronic conductivity, $E_{\text {a }}$ activation energy, $k$ the Boltzmann constant, $T$ the temperature, $p\left(\mathrm{O}_{2}\right)$ the partial pressure of gaseous oxygen and $n$ a value that depends on the nature of the point defects arising when oxygen is removed from the lattice.

The reactions occurring on the surface of $\mathrm{TiO}_{2}$ gas sensor can be summarized as

$$
\begin{aligned}
& e^{-}+\mathrm{O}_{2} \stackrel{T<500 \mathrm{~K}}{\longrightarrow} \mathrm{O}_{2}^{-} \\
& e^{-}+\mathrm{O}_{2}^{-} \stackrel{T>500 \mathrm{~K}}{\longrightarrow} 2 \mathrm{O}^{-} \\
& \mathrm{C}_{\mathrm{n}} \mathrm{H}_{2 \mathrm{n}+2}+2 \mathrm{O}^{-} \longleftrightarrow \mathrm{H}_{2} \mathrm{O}+\mathrm{C}_{\mathrm{n}} \mathrm{H}_{2 \mathrm{n}} \mathrm{O}+e^{-},
\end{aligned}
$$

where $\mathrm{C}_{\mathrm{n}} \mathrm{H}_{2 \mathrm{n}+2}$ represents various hydrocarbons.

The first two reactions takes place in air, due to which carrier concentration is low and thus, increasing the resistance. The last reaction corresponds to oxidation of reducing carriers. This increases the carrier concentration and hence decreases the resistance on exposing to reducing gases.

\section{Conclusions}

Earlier investigators have not made experimentations for titania at room temperature; they have investigated it at other higher temperatures. Therefore, the research in this paper has demonstrated the possibility and potential of a nanosized $\mathrm{TiO}_{2}$-based sensor operable at room temperature for sensing of LPG. SEM micrographs show a cluster like morphology with highly porous structure. From XRD, minimum crystallite size was $30 \mathrm{~nm}$. The average sensitivity of sensor was 1.7. Rapid sensor response, good stability and satisfactory sensitivity demonstrate the promise of this sensor for LPG determination in the industrial and environmental monitoring.

\section{Acknowledgements}

Financial support from UGC as Major Research Project F. No. 36-265/2008 (SR) is highly acknowledged. 


\section{References}

Barsan N, Koziej D and Weimar U 2007 Sensors \& Actuators B121 18

Baruwati B, Kumar D K and Manorama S V 2006 Sensors \& Actuators B119 676

Birkefeld L D, Azad A M and Akbar S A 1992 J. Am. Ceram. Soc. 752964

Bonini N, Carotta M C, Chiorino A, Guidi V, Malagù C, Martinelli G, Paglialonga L and Sacerdoti M 2000 Sensors \& Actuators B68 274

Cabot A, Arbiol J, Morante J R, Weimar U, Barsan N and Gopal W 2000 Sensor \& Actuators B70 87

Carney C M, Yoo S and Sheikh A A 2005 Sensors \& Actuators B108 29

Chen Y J, Zhu C L and Xio G 2008 Sensors \& Actuators B129 639

Coronado J M, Kataoka S, Tejedor T and Anderson M A 2003 J. Catal. 219219

Devi G S, Hyodo T, Shimizu Y and Egashira M 2002 Sensors \& Actuators $\mathbf{B 8 7} 122$

Dhawale D S, Salunkhe R R, Patil U M, Gurav K V, More A M and Lokhande C D 2008 Sensor \& Actuators B134 988

Faramarz H B, Kesmhiri M, Kakavand M and Troczynski T 2005 Sensors \& Actuators B110 28

Guidi V, Carotta M C, Ferroni M, Martinelli G, Paglialonga L, Comini E and Sberveglieri G 1999 Sensors \& Actuators $\mathbf{B 5 7}$ 197

Kirner U, Schierbaum K D, Gopal W, Leibold B, Nicoloso N, Weppner W, Fischer D and Chu W F 1990 Sensors \& Actuators B1 103

Kitamura T and Yokoyama M 1991 J. Appl. Phys. 69821
Lee K, Lee N H, Shin S H and Kim S J 2000 Mater. Sci. Eng. B129 109

Li M and Chen Y 1996 Sensors \& Actuators B32 83

Liu Z, Yamazaki T, Shen Y, Kikuta T, Nakatani N and Li Y 2008 Sensors \& Actuators B129 666

Manera M G, Cozzoli P D, Leo G, Curri M L, Agostiano A, Vasanelli L and Rella R 2007 Sensors \& Actuators B126 562

Micheli A L 1984 Am. Cer. Soc. Bull. 63694

More A M, Gunjakar J L and Lokhande C D 2008 Sensors \& Actuators B129 671

More P, Kumar R, Yadav B C and Khanna P K 2009 Inter. J. Green Nanotechnol.: Mater. Sci. Eng. $1 \mathrm{M} 3$

Ruiz A M, Cornet A, Shimanoe K, Morante J R and Yamazoe N 2005 Sensors \& Actuators B108 34

Shinde V R, Gujar T P and Lokhande C D 2007 Sensors \& Actuators B123 701

Srivastava A, Jain K, Rashmi, Srivastava A K and Lakshmikumar S T 2006 Mater. Chem. Phys. 9785

Thiagarajan S, Su B W and Chen S M 2009 Sensors \& Actuators B136 464

Wu Y 2009 Sensors \& Actuators B137 80

Yadav B C, Srivastava A K and Sharma P 2007 Sensor \& Transducers 1811348

Yadav B C, Srivastava R, Dwivedi C D and Pramanik P 2008a Sensors \& Actuators B131 216

Yadav B C, Srivastava R, Yadav A and Srivastava V 2008b Sensor Letts. 6714

Yadav B C, Srivastava R and Yadav A 2009a Sensors \& Materials 2187

Yadav B C, Yadav A, Shukla T and Singh S 2009b Sensor Letts. 71 